\title{
Right-handed neutrinos: Dark matter, lepton flavor violation, and leptonic collider searches
}

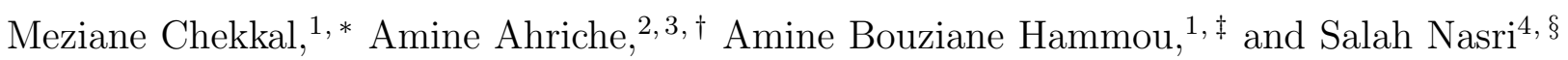 \\ ${ }^{1}$ Department of Physics, University of Sciences and Technology of Oran, \\ B.P. 1505, Oran, El M'Naouer, Algeria \\ ${ }^{2}$ Department of Physics, University of Jijel, \\ P.B. 98 Ouled Aissa, DZ-18000 Jijel, Algeria \\ ${ }^{3}$ The Abdus Salam International Centre for Theoretical Physics, \\ Strada Costiera 11, I-34014, Trieste, Italy \\ ${ }^{4}$ Department of Physics, United Arab Emirates University, \\ P.O. Box 15551, Al-Ain, United Arab Emirates
}

\begin{abstract}
We examine lepton flavor violating (LFV) interactions for heavy right-handed neutrinos that exist in most of the standard model extensions that address dark matter (DM) and neutrino mass at the loop level. In order to probe the collider effect of these LFV interactions, we impose the assumption that the model parameters give the right values of the DM relic density and fulfill the constraints from the LFV processes $\ell_{\alpha} \rightarrow \ell_{\beta} \gamma$ and $\ell_{\alpha} \rightarrow 3 \ell_{\beta}$. We also investigate the possibility of probing these interactions, and hence the right-handed neutrino, at leptonic colliders through different final state signatures.
\end{abstract}

\footnotetext{
*Electronic address: meziane.chekkal@univ-usto.dz, meziane.chekkal@gmail.com

$\dagger$ Electronic address: aahriche@ictp.it, aahriche@gmail.com

${ }^{\ddagger}$ Electronic address: amine.hammou@univ-usto.dz, hbamine@gmail.com

$\S$ Electronic address: snasri@uaeu.ac.ae
} 


\section{INTRODUCTION}

The standard model (SM) of particle physics describes the properties of elementary particles and their interactions and has been in good agreement with numerous experiments, so far. Moreover, one of its last building blocks, namely, the Higgs boson, has been detected, and there are future planed experiments to precisely measure its couplings to matter as well as its selfcoupling. Neutrino oscillation data established that at least two of the three SM neutrinos have mass and nonzero mixing. However, their properties, such as their nature and the origin of the smallness of their mass, have no explanation within the SM, which cry out for new physics. One of the most popular mechanisms for understanding why neutrino mass is tiny is the seesaw mechanism [1], which assumes the existence of right-handed $(\mathrm{RH})$ neutrinos with masses several orders of magnitude heavier than the electroweak (EW) scale. In this approach, the dimension five operator $(L \Phi)^{2} / \Lambda$ will be induced in the low-energy effective Lagrangian, with $\Lambda$ the scale of the new physics which is of the order of the RH neutrino mass scale. Unfortunately, such heavy particles decouple from the low-energy spectrum at energies many orders of magnitude higher than the EW scale and so cannot be directly probed in high-energy physics experiments. Even in a low-scale seesaw mechanism where $\Lambda \sim \mathrm{TeV}$, one typically expects the active-singlet neutrino mixing to be smaller than $10^{-5}$, making the production cross section well below the current and the near future collider sensitivities [2]. Moreover, for a RH neutrino heavier than $10^{7} \mathrm{GeV}$, it can destabilize the electroweak vacuum via the large loop corrections induced by the Dirac neutrino Yukawa term.

Another possible way to explain the smallness of neutrino masses is by generating them at the loop level [3 7]. In this mechanism, the tree-level neutrino mass term vanishes due to some discrete symmetry and can be induced via loop diagrams with a loop suppression factor, and, consequently, the scale of new physics $\Lambda$ can be much lower than the conventional seesaw mechanism. For instance, as it has been shown in Ref. [8], the scale of new physics can be in the sub-TeV for the three-loop neutrino mass generation model [6], which makes it testable at collider experiments [9].

In most of the radiative neutrino mass models, neutrinos are Majorana particles, and so one expects a violation of the total lepton number. This will have stringent phenomenological implications on loop-induced neutrino mass models and can be a direct indication of new physics. Indeed, lepton flavor violating (LFV) processes are currently the object of great attention; the experiments which aim at detecting them are becoming increasingly precise and clean. The radiative decay $\mu \rightarrow e \gamma$ is simplest to detect; indeed, only one particle is created in the finale state, the electron. Its energy is thus of the order of $m_{\mu}=105 \mathrm{MeV}$, which is far from the principal background $\mu \rightarrow e \overline{\nu_{e}} \nu_{\mu}$ disintegration, whose energy spectrum decreases drastically beyond $m_{\mu} / 2$. The current experimental limits for these low-energy processes are $\mathcal{B}(\mu \rightarrow$ e $\gamma)<5.7 \times 10^{-13}$ $\left(\mathrm{MEG}\right.$ [10]), $\mathcal{B}\left(\mu \rightarrow e^{-} e^{+} e^{-}\right)<10^{-12}$ (SINDRUM [11]), $\mathcal{B}(\tau \rightarrow e \gamma)<3.3 \times 10^{-8}(B A B A R[12])$, $\mathcal{B}\left(\tau \rightarrow e^{-} e^{+} e^{-}\right)<2.7 \times 10^{-8}$ (BELLE [13]), $\mathcal{B}(\tau \rightarrow \mu \gamma)<4.4 \times 10^{-8}$ (BABAR [12]) and $\mathcal{B}\left(\tau \rightarrow \mu^{-} \mu^{+} \mu^{-}\right)<2.1 \times 10^{-8}$ (BELLE [13]), and some of these bounds are expected to improve by almost an order of magnitude in the next couple of years. In particular, the MEG experiment will be sensitive to a $\mu \rightarrow e \gamma$ branching ratio as low as $6 \times 10^{-14}$ which will be able to strongly constrain a large class of radiative neutrino mass models.

In this work, we study the phenomenological implications of a class of radiative neutrino mass models based on the SM extension by RH neutrinos and a singlet charged scalar and in which the lightest $\mathrm{RH}$ neutrino is a dark matter candidate. After imposing the current bounds from the LFV processes on the model parameters, we determine the parameters space for which the 
$\mathrm{RH}$ relic density is in agreement with the observation. We also consider the constraint on singleand multiphoton events with missing energy reported by the L3 Collaboration at LEP [14]. In our scan, we find that many of the benchmark points that satisfy the LFV constraints require a cancellation among the product combinations of the coupling to cancel out. For that, we define a quantify that quantifies such a fine-tuning parameter and use it to classify the viable model parameter space. Finally, we study the possibility of observing a signal at future lepton colliders.

The paper is organized as follows: In Sec. II, we present a class of interactions involving right-handed neutrinos, that appear in many radiative neutrino mass models, and discuss the constraints from the different LFV processes. In Sec. III, we show that the lightest RH neutrino can be a viable dark matter candidate while satisfying the current experimental bounds on LFV processes. In Sec. IV, we analyze the monophoton events with missing energy using the data collected by the L3 Collaboration at LEP-II and determine the viable parameter space for such models. In Sec. V, we consider three benchmark points (according to the fine-tuning degree) and study the possible signature of the RH neutrino and charged scalar signals at lepton colliders. We also discuss the effect of using polarized beams on the signal significance. Finally, we give our conclusion in Sec. VII.

\section{LFV CONSTRAINS CLASS OF MODELS WITH RIGHT-HANDED NEUTRINOS}

We consider a class of radiative neutrino mass models based on extending the SM with three right-handed neutrinos $N_{i}(i=1,2,3)$ and a charged scalar field $S^{ \pm}$which is an $S U(2)_{L}$ singlet. For the purpose of our study, the relevant term in the Lagrangian has the form [5] 7, 15]

$$
\mathcal{L}_{\mathcal{N}} \supset-\frac{1}{2} m_{N_{i}} \overline{N_{i}^{c}} P_{R} N_{i}+g_{i \alpha} S^{+} \overline{N_{i}} \ell_{\alpha_{R}}+\text { H.c. }
$$

where $\ell_{\alpha_{R}}$ is the right-handed charged lepton and $g_{i \alpha}$ are Yukawa couplings. The global $Z_{2}$ symmetry is imposed ${ }^{1}$ to ensure the stability of the lightest $\mathrm{RH}$ neutrino, that is supposed to play the dark matter $(\mathrm{DM})$ role. These interactions can give rise to the LFV processes $\ell_{\alpha} \rightarrow \ell_{\beta} \gamma(\{\alpha=\mu, \beta=e\})$ and $\ell_{\alpha} \rightarrow 3 \ell_{\beta}(\{\alpha=\tau, \beta=e, \mu\})$, that are mediated by the $\mathrm{RH}$ neutrinos and the charged scalar fields as shown in Figs. 1 and 2 , respectively.

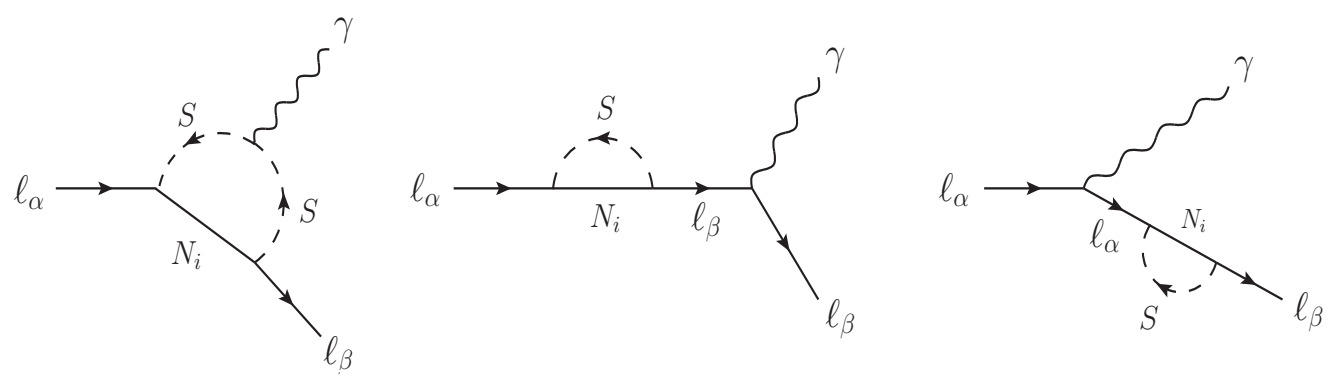

FIG. 1: The one-loop diagrams that contribute to $\ell_{\alpha} \rightarrow \ell_{\beta} \gamma$.

The contribution of the interactions (1) to the branching ratio $\ell_{\alpha} \rightarrow \ell_{\beta} \gamma$ is [17]

${ }^{1}$ In some neutrino mass models like Ref. [16], where the RH neutrino are promoted to higher representations (septplet), the global $Z_{2}$ symmetry is accidental. 

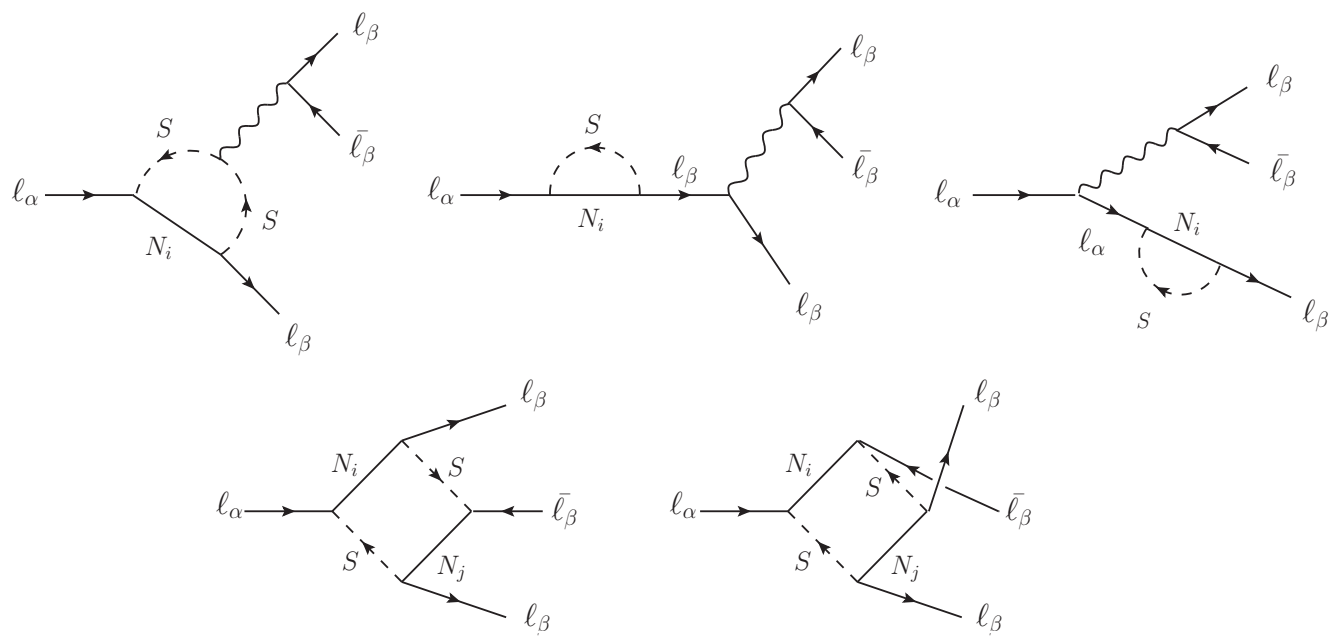

FIG. 2: The diagrams contributing to $\ell_{\alpha} \rightarrow 3 \ell_{\beta}$. The penguin (box) diagrams are shown in the top (bottom).

$$
\mathcal{B}^{(N)}\left(\ell_{\alpha} \rightarrow \ell_{\beta} \gamma\right)=\frac{3(4 \pi)^{3} \alpha}{4 G_{F}^{2}}\left|A_{D}\right|^{2} \times \mathcal{B}\left(\ell_{\alpha} \rightarrow \ell_{\beta} \nu_{\alpha} \bar{\nu}_{\beta}\right)
$$

where $G_{F}$ is the Fermi constant, $\alpha=e^{2} / 4 \pi$ is the electromagnetic fine structure constant, and $A_{D}$ is the dipole contribution that is given by

$$
A_{D}=\sum_{i=1}^{3} \frac{g_{i \beta}^{*} g_{i \alpha}}{2(4 \pi)^{2}} \frac{1}{m_{S}^{2}} F\left(x_{i}\right),
$$

with $x_{i}=m_{N_{i}}^{2} / m_{S}^{2}$ and $F(x)$ is a loop function given in the Appendix.

For $\ell_{\alpha}=\ell_{\beta}=\mu$, Fig. 1 represents a new contribution to the muon anomalous magnetic moment $\delta a_{\mu}$, and it is given by

$$
\delta a_{\mu}^{(N)}=\frac{1}{16 \pi^{2}} \frac{m_{\mu}^{2}}{m_{S}^{2}} \sum_{i}\left|g_{i \mu}\right|^{2} F_{2}\left(\frac{m_{N_{i}}^{2}}{m_{S}^{2}}\right) .
$$

The branching ratio for $\ell_{\alpha} \rightarrow \ell_{\beta} \bar{\ell}_{\beta} \ell_{\beta}$ is [17]

$$
\begin{aligned}
\mathcal{B}^{(N)}\left(\ell_{\alpha} \rightarrow \ell_{\beta} \bar{\ell}_{\beta} \ell_{\beta}\right) & =\frac{3(4 \pi)^{2} \alpha^{2}}{8 G_{F}^{2}}\left[\left|A_{N D}\right|^{2}+\left|A_{D}\right|^{2}\left(\frac{16}{3} \log \left(\frac{m_{\alpha}}{m_{\beta}}\right)-\frac{22}{3}\right)\right. \\
& +\frac{1}{6}|B|^{2}+\frac{1}{3} \frac{m_{\alpha}^{2} m_{\beta}^{2}\left(3 \sin ^{4} \theta_{W}-\sin ^{2} \theta_{W}+\frac{1}{4}\right)}{m_{W}^{4} \sin ^{4} \theta_{W}}\left|A_{D}\right|^{2} \\
& \left.+\left(-2 A_{N D} A_{D}^{*}+\frac{1}{3} A_{N D} B^{*}-\frac{2}{3} A_{D} B^{*}+\text { h.c. }\right)\right] \\
& \times \mathcal{B}\left(\ell_{\alpha} \rightarrow \ell_{\beta} \nu_{\alpha} \bar{\nu}_{\beta}\right) .
\end{aligned}
$$

Here $\theta_{W}$ is the Weinberg mixing angle. The coefficients $A_{N D}$ and $B$ are the nondipole contribution from the photonic penguin and the box diagrams, respectively, which read 


$$
A_{N D}=\sum_{i=1}^{3} \frac{g_{i \beta}^{*} g_{i \alpha}}{6(4 \pi)^{2}} \frac{1}{m_{S}^{2}} G\left(x_{i}\right)
$$

and

$$
B=\frac{1}{(4 \pi)^{2} e^{2} m_{S}^{2}} \sum_{i, j=1}^{3}\left[\frac{1}{2} D_{1}\left(x_{i}, x_{j}\right) g_{j \beta}^{*} g_{j \beta} g_{i \beta}^{*} g_{i \alpha}+\sqrt{x_{i} x_{j}} D_{2}\left(x_{i}, x_{j}\right) g_{j \beta}^{*} g_{j \beta}^{*} g_{i \beta} g_{i \alpha}\right],
$$

where $G(x), D_{1}\left(x_{i}, x_{j}\right)$, and $D_{2}\left(x_{i}, x_{j}\right)$ are loop functions given in the Appendix.

The contribution of the photonic dipole term is present in both branching ratios and is more important than the photonic nondipole term regardless of the values of the couplings and the $\mathrm{RH}$ neutrinos and the charged scalar masses. It is worth pointing out that it is possible to chose the model parameters for which the branching ratio of the trilepton channel is larger than the one for the $\ell_{\alpha} \rightarrow \ell_{\beta} \gamma$ channel where the main contribution comes from the box diagrams in Fig. 2 .

We perform a numerical scan over all free parameters of the model to probe possible signatures of new physics at colliders. In addition to the LFV constraints, we require the Yukawa couplings $g_{i \alpha}$ to be perturbative. In order to avoid the bounds on $\ell_{\alpha} \rightarrow \ell_{\beta}+\gamma$, one has to consider a small value for $g_{i \alpha}$ or have a cancellation between the different terms in the expression of $A_{D}$ (3). To quantify the fine-tuning that ensures such cancellation, we define

$$
R=\frac{\left|\sum_{i=1}^{3} g_{i \beta}^{*} g_{i \alpha} F\left(x_{i}\right)\right|^{2}}{\operatorname{Max}\left[\left|g_{i \beta}^{*} g_{i \alpha} F\left(x_{i}\right)\right|^{2}\right]},
$$

which we will call the fine-tuning parameter. In this case, very small $R$ corresponds to a severe tuning on the model parameters. This could allow the Yukawa coupling $g_{i \alpha}$ to be large, which is interesting for collider searches. In what follows, we consider the fine-tuning parameter only for the process $\mu \rightarrow e+\gamma$ (i.e., $\alpha=\mu$ and $\beta=e$ ), since this is the most severely constrained.

In Fig. 33, we present the branching ratios for the processes $\ell_{\alpha} \rightarrow \ell_{\beta} \gamma, \ell_{\alpha} \rightarrow 3 \ell_{\beta}$, and the anomalous magnetic moment of the muon as a function of the charged scalar mass for different values of the ratio $R \approx 1,10^{-2}, 10^{-4}$. We note that although the most stringent constraint on LFV from the process $\mu \rightarrow e \gamma$ can be fulfilled for $R_{1} \approx 1$ by taking the couplings $g_{i e}$ and $g_{i \mu}$ small, this may be in conflict with the DM relic density for some values of the charged scalar masses, whereas for $R_{2} \approx 10^{-2}$ and $R_{3} \approx 10^{-4}$ the choice of ratio has a minor impact on the LFV branching fractions of the muon and tau decay processes.

As can be seen, for scalar and the RH neutrinos lighter than few hundreds GeV, the LFV decay processes can be in agreement with the experimental bounds. For the muon anomalous magnetic moments, the Yukawa interaction terms $S \bar{N} \ell_{R}$ give a contribution smaller than $10^{-12}$, and, hence, does not account for the $3.6 \sigma$ deviation from the SM prediction [18], and require new physics if the discrepancy will be confirmed at the $5 \sigma$ level by the upcoming experiments [19].

In this class of models, the interactions term (1) does not induce a contribution to neutrinoless double beta decay, since both $N_{R}$ and $S^{ \pm}$do not couple to the quarks. However, if these interactions are embedded within a larger gauge symmetry, such as left-right symmetric models $\left[S U(2)_{L} \times S U(2)_{R}\right]\left[20\right.$, then there will be a contribution which involves the $W_{R}^{ \pm}$and depends on $M_{W_{R}}, m_{N}$, and the new gauge coupling strength. Depending on the model details, when light Majorana neutrino masses $m_{\alpha \beta}^{(\nu)}$ are generated, there will be contributions proportional to the element $m_{e e}^{(\nu)}$, for which the bound on the rate of $0 \nu \beta \beta$ can be easily satisfied. 

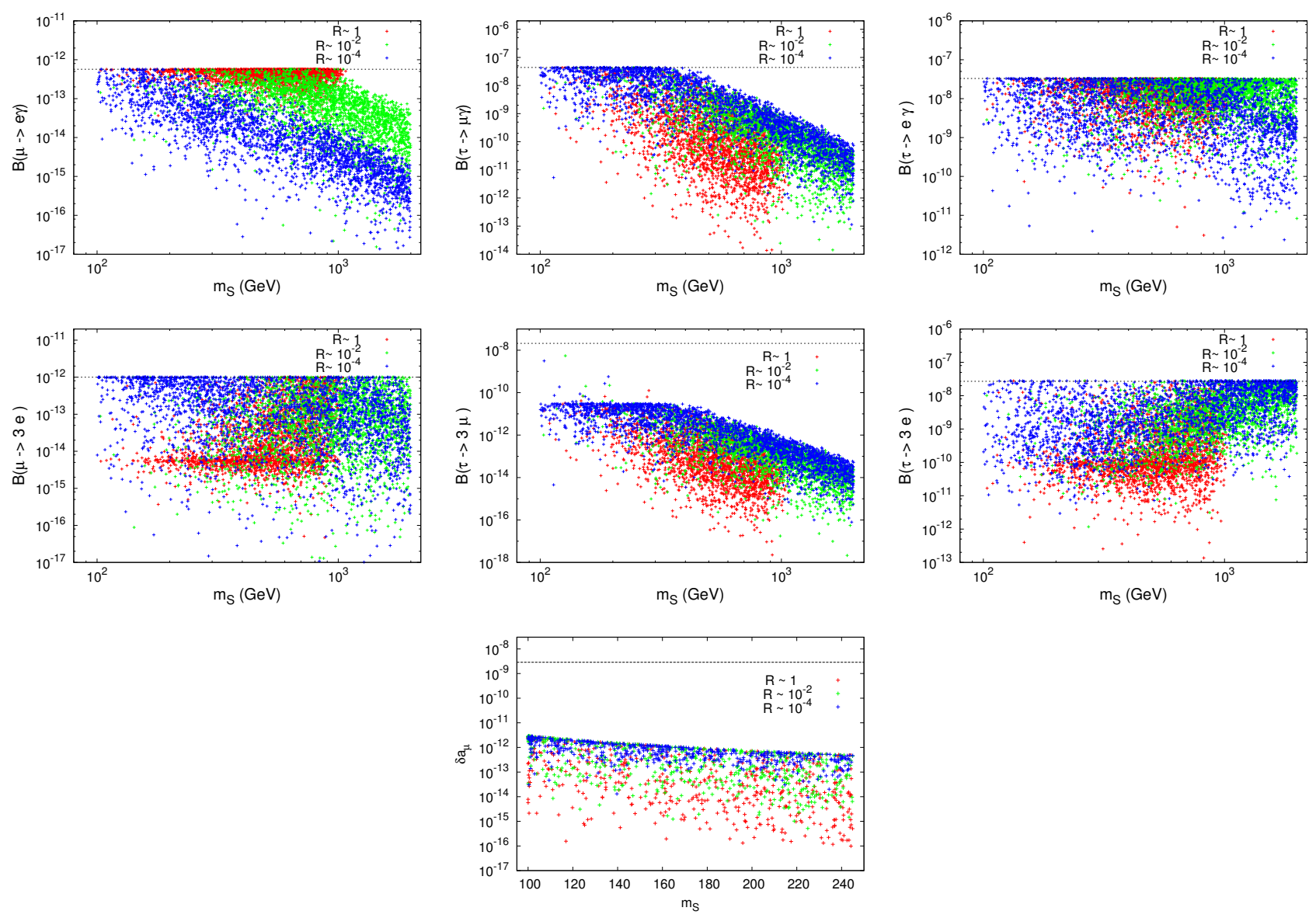

FIG. 3: The branching ratios (top) $\mathcal{B}(\mu \rightarrow e \gamma), \mathcal{B}(\tau \rightarrow \mu \gamma)$ and $\mathcal{B}(\tau \rightarrow e \gamma)$; and (middle) $\mathcal{B}(\mu \rightarrow 3 e)$, $\mathcal{B}(\tau \rightarrow 3 \mu)$, and $\mathcal{B}(\tau \rightarrow 3 e)$ as a function of $m_{S}$. Some hundreds of configurations of free parameters satisfying the experimental data are used. In the bottom, we show the contribution to the muon anomalous magnetic moment. The horizontal dashed lines show the current experimental upper bounds for these observables.

\section{DARK MATTER: RELIC DENSITY}

As mentioned earlier, the lightest RH neutrino $N_{1}$ is stable and could be a good DM candidate. In the hierarchical RH neutrino mass spectrum case, we can safely neglect the effect of $N_{2}$ and $N_{3}$ for $N_{1}$ density. The $N_{1}$ number density gets depleted through the annihilation process $N_{1} N_{1} \rightarrow \ell_{\alpha} \ell_{\beta}$ via $t$-channel exchange of $S^{ \pm}$. For two incoming DM particles with momenta $p_{1}$ and $p_{2}$, and final state charged leptons with momenta $k_{1}$ and $k_{2}$, the amplitude is given by

$$
\mathcal{M}_{\alpha \beta}=g_{1 \alpha} g_{1 \beta}^{*}\left[\frac{\bar{u}\left(k_{1}\right) P_{L} u\left(p_{1}\right) \cdot \bar{v}\left(p_{2}\right) P_{R} v\left(k_{2}\right)}{t-m_{S}^{2}}-\frac{\bar{u}\left(k_{1}\right) P_{L} u\left(p_{1}\right) \cdot \bar{v}\left(p_{2}\right) P_{R} v\left(k_{2}\right)}{u-m_{S}^{2}}\right],
$$

where $t=\left(p_{1}-k_{1}\right)^{2}$ and $u=\left(p_{1}-k_{1}\right)^{2}$ are the Mandelstam variables corresponding to the $t$ and $u$ channels, respectively. After squaring, summing, and averaging over the spin states, we 
find that, in the nonrelativistic limit, the total annihilation cross section reads ${ }^{2}$

$$
\sigma_{N_{1} N_{1}} v_{r} \simeq \sum_{\alpha, \beta}\left|g_{1, \alpha} g_{1, \beta}^{*}\right|^{2} \frac{m_{N_{1}}^{2}\left(m_{S}^{4}+m_{N_{1}}^{4}\right)}{48 \pi\left(m_{S}^{2}+m_{N_{1}}^{2}\right)^{4}} v_{r}^{2}
$$

with $v_{r}$ the relative velocity between the annihilating $N_{1}$ particles. As the temperature of the Universe drops below the freeze-out temperature $x_{f}=m_{N_{1}} / T_{f} \approx 25$, the annihilation rate becomes smaller than the expansion rate (the Hubble parameter) of the Universe, and the $N_{1}$ 's start to decouple from the thermal bath. The relic density after the decoupling can be obtained by solving the Boltzmann equation, which approximately yields [22]

$$
\Omega_{N_{1}} h^{2} \simeq \frac{2 x_{f} \times 1.1 \times 10^{9} \mathrm{GeV}^{-1}}{\sqrt{g^{*}} M_{p l}\left\langle\sigma_{N_{1} N_{1}} v_{r}\right\rangle} \simeq \frac{17.56}{\sum_{\alpha, \beta}\left|g_{1 \alpha} g_{1 \beta}^{*}\right|^{2}}\left(\frac{m_{N_{1}}}{50 \mathrm{GeV}}\right)^{2} \frac{\left(1+m_{S}^{2} / m_{N_{1}}^{2}\right)^{4}}{1+m_{S}^{4} / m_{N_{1}}^{4}},
$$

where $\left\langle v_{r}^{2}\right\rangle \simeq 6 / x_{f} \simeq 6 / 25$ is the thermal average of the relative velocity squared of a pair of two $N_{1}$ particles, $M_{p l}$ is the Planck mass, and $g_{*}\left(T_{f}\right)$ is the total number of effective massless degrees of freedom at the freeze-out.

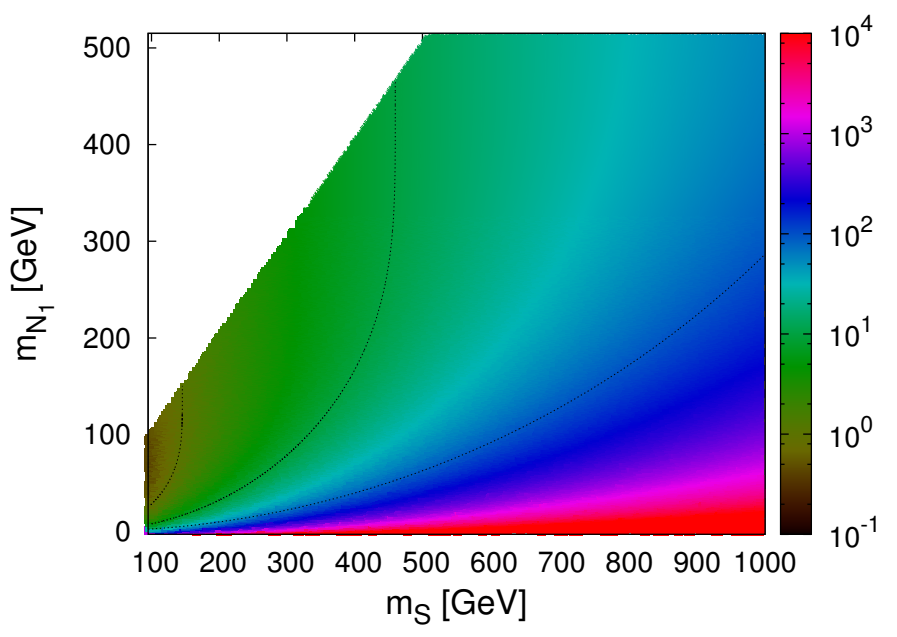

FIG. 4: Dark matter mass versus the charged scalar mass, where in the palette one reads the coupling combination $\sum_{\alpha \beta}\left|g_{1 \alpha} g_{1 \beta}^{*}\right|^{2}$, that enters in the expression of the relic density and can affect the rates of the LFV processes. The dashed curves (from left to right) represent the values $\sum_{\alpha \beta}\left|g_{1 \alpha} g_{1 \beta}^{*}\right|^{2}=$ $1,10,100$, respectively.

To see the impact of the DM relic density on the model parameters, we present in Fig. 4 the quantity $\sum_{\alpha \beta}\left|g_{1 \alpha} g_{1 \beta}^{*}\right|^{2}$ as a palette in the contours $m_{N_{1}}$ versus $m_{S}$ within the conditions $m_{N_{1}}<m_{S}$ and $m_{S}>100 \mathrm{GeV}$ being imposed. For values of $\sum_{\alpha \beta}\left|g_{1 \alpha} g_{1 \beta}^{*}\right|^{2}$ larger than 10 (corresponding to the light-greenish blue color in the palette), it is difficult to maintain all LFV process branching ratios within the current experimental limits, and an extreme fine-tuning is required. On the other hand, the heavier $N_{1}$ is, the more restricted the allowed range of the

${ }^{2}$ In some models like Ref. [21], there are some annihilation channels beside $N_{1} N_{1} \rightarrow \ell_{\alpha} \ell_{\beta}$. In this case, the annihilation cross section of $N_{1} N_{1} \rightarrow \ell_{\alpha} \ell_{\beta}$ should be smaller than $\sqrt{10}$, and the combination $\sum_{\alpha, \beta}\left|g_{1, \alpha} g_{1, \beta}^{*}\right|^{2}$ should be less than its value in (11). 
charged scalar mass is. Therefore, the most viable range of the masses that are consistent with both the DM relic density and the current bounds on LFV processes are $m_{N_{1}}<200 \mathrm{GeV}$ and $m_{S}<300 \mathrm{GeV}$ while keeping $m_{N_{1}}<m_{S}$.

With regard to the constraint from the DM direct detection experiments, since the interactions of $N_{1}$ involve only a charged lepton, the DM-nucleus scattering is absent at the tree level ${ }^{3}$, and cannot be induced at one loop via the exchange of a photon, since for a Majorana particle the magnetic moment vanishes identically. But if the next lightest $\mathrm{RH}$ neutrino, say, $N_{2}$, is quasidegenerate with a mass splitting of the order or less than a few $\mathrm{keV}$, then the inelastic scattering $N_{1}+$ nuclues $\rightarrow N_{2}$ +nucleus is possible. However, such a situation is highly unnatural for such a tiny mass splitting to be stable under a radiative correction which can render the scattering kinematically forbidden.

\section{CONSTRAINTS FROM LEP-II}

Here we consider the analysis of single- and multiphoton events with missing energy by the L3 detector at LEP, for center-of-mass energies between 189 and $209 \mathrm{GeV}$. It was found that the cross section of the process $e^{+} e^{-} \rightarrow \nu \bar{\nu} \gamma(\gamma)$ is in agreement with the SM expectations, and there was no evidence for a massive neutral particle with a significance higher than three that can be produced at LEP-II. This result can have a significant impact on the parameter space relevant for DM and neutrino oscillation data. Thus, we confront thousands of randomly generated benchmark points that respect the different DM and LFV constraints together with the LEP-II data. As a subsequent study of the electron-positron (electron-electron) collision on the International Linear Collider (ILC) will be carried out in the next sections, it is necessary to sort out the benchmark points of $N_{2}$ and $N_{3}$ based on whether their decay via a three-body process will occur inside or outside the detector. In Fig. 5, we present the decay length for $N_{2}$ and $N_{3}$, where one can see that $N_{3}$ decay mostly inside the detector, whereas an appreciable fraction of $N_{2}$ events escape from the detector. Consequently, only $N_{1}$ and the $N_{2}$ events that decay outside the detector will be counted as missing energy and, hence, are subject to the LEP constraint. In all our analyses, we apply this selection criterion.

${ }^{3}$ If the mass of $N_{1}$ arises from the vacuum expectation value of some singlet scalar field, then a low-energy effective operator of the form $\bar{q} q N_{1} N_{1}$ will be generated at the tree level (see the last reference in Ref. [8]). 

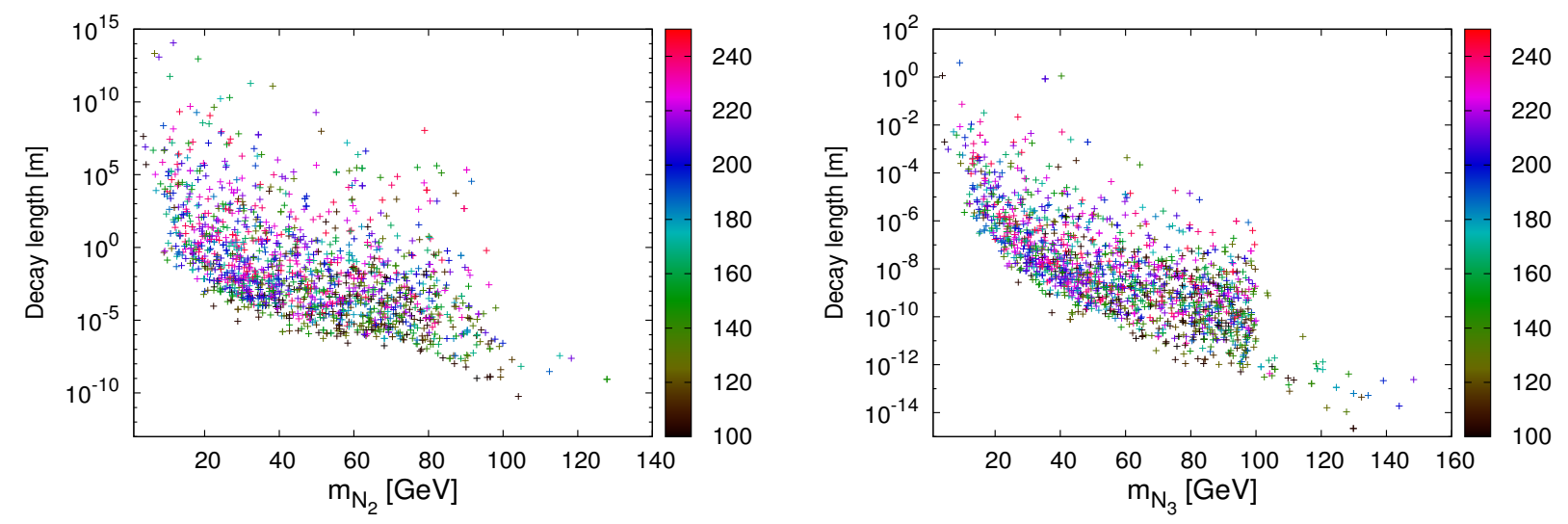

FIG. 5: The decay length of the RH neutrinos $N_{2}$ (left) and $N_{3}$ (right) as a function of $m_{N_{2}}$ and $m_{N_{3}}$, respectively. The palette represents the charged scalar mass $m_{S}[\mathrm{GeV}]$.

We consider the center-of-mass energies $\sqrt{s}=188.6 \mathrm{GeV}$ and $\sqrt{s}=207 \mathrm{GeV}$ at the highest integrated luminosities 176 and $130.2 \mathrm{pb}^{-1}$, respectively. In order to increase the signal significance, we apply the following kinematical cuts used by the L3 Collaboration to look for a high-energy single photon [14]:

- the polar angle of the photon: $\left|\cos \theta_{\gamma}\right|<0.97$,

- the transverse momentum of the photon: $p_{t}^{\gamma}>0.02 \sqrt{s}$, and

- the energy of the photon: $E_{\gamma}>1 \mathrm{GeV}$.

Using the LANHEP/CCALCHEP packages [23, 24, to which the interaction terms in (1) are implemented, we compute the cross sections of the signal $e^{-} e^{+} \rightarrow \gamma+E_{\text {miss }}$ and the background $e^{-} e^{+} \rightarrow \nu_{i} \bar{\nu}_{j} \gamma$ for the aforementioned benchmark points. Furthermore, we evaluate the significance at the corresponding luminosity as a function of the dark matter mass. Moreover, we found that the cross section is sensitive to the inverse of $m_{S} m_{N_{i}}$ as well products of the interaction couplings, and hence we present a combination of these parameters. This lead us to derive an exclusion bound on a combination of these parameters.

The results are shown in Fig. 6, requiring that at such energies and the corresponding luminosity values the significance $S$ must be smaller than three $(S<2$ for a conservative choice) leads to a constraint on the model parameters $\mathrm{as}^{4}$

$$
\Delta=\sum_{i, k}\left|g_{i e} g_{k e}^{*}\right|^{2}\left[\frac{150 \mathrm{GeV}}{m_{S}}\right]\left[\frac{50 \mathrm{GeV}}{\sqrt{m_{N_{i}} m_{N_{k}}}}\right]<1.95
$$

where the summation is performed over $\mathrm{RH}$ neutrinos that contribute to the missing energy, to which according to Fig. 5 only $N_{1}$ and $N_{1}$ contribute. From the large values of $\Delta$ in the palette, one concludes that the absence of new physics at LEP can put a significant constraint on the strength of the interaction $S^{+} \overline{N_{i}} \ell_{\alpha_{R}}$, especially when the $\mathrm{RH}$ neutrino is lighter than $50 \mathrm{GeV}$. Consequently, it can have an important impact on the scale of the generated neutrino mass in models based on such a type of interaction.

${ }^{4}$ For $S<2$ the constraint 12 becomes $\sum_{i, k}\left|g_{i e} g_{k e}^{*}\right|^{2}\left[\frac{150 \mathrm{GeV}}{m_{S}}\right]\left[\frac{50 \mathrm{GeV}}{\sqrt{m_{N_{i}} m_{N_{k}}}}\right]<1.65$. 

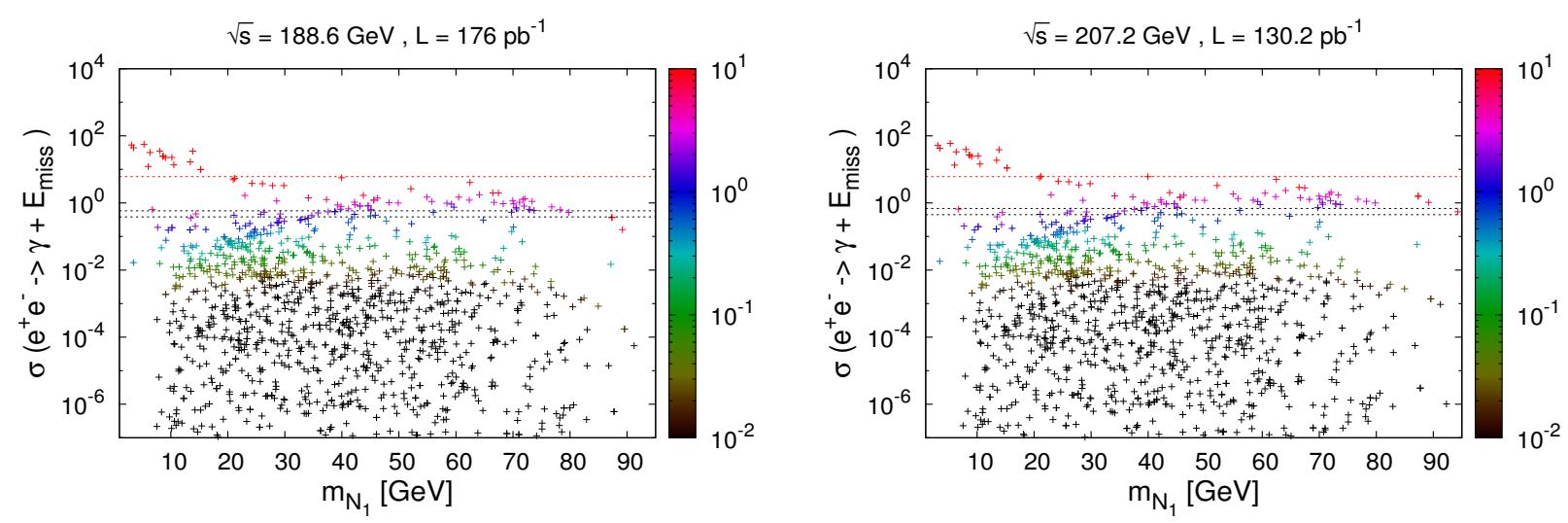

FIG. 6: The cross section for the randomly chosen benchmark points for the process $e^{-} e^{+} \rightarrow \gamma+E_{\text {miss }}$ at $\mathrm{LEP}$ as a function of $m_{N_{1}}$ for the $\mathrm{CM}$ energies $\sqrt{s}=188.6 \mathrm{GeV}$ (right) and $\sqrt{s}=207.2 \mathrm{GeV}$ (left). The palette represents the combination $\Delta$, and the black dashed lines correspond to $S=2$, 3 , respectively. The red dashed line corresponds to the background.

\section{POSSIBLE SIGNATURES AT LEPTON COLLIDERS}

The study of an electron-positron and/or electron-electron collision at lepton colliders such as the ILC [25] represents a new approach for probing/detecting new physics in the tera scale. The ILC is designed to cover center-of-mass (CM) energies from 250 to $500 \mathrm{GeV}$, with the possibility to expand it up to $1 \mathrm{TeV}$ and with the option of using polarized beams for both electrons and positrons. Another lepton collider which is under development is the Compact Linear Collider (CLIC) which will provide high-luminosity $e^{+} e^{-}$collisions with $\mathrm{CM}$ energy from $380 \mathrm{GeV}$ to $3 \mathrm{TeV}$ [26]. Here, in this work, we consider the main processes where the DM particles are in the final states via the interactions in (1) and could induce an excess in the event number relative to the SM background.

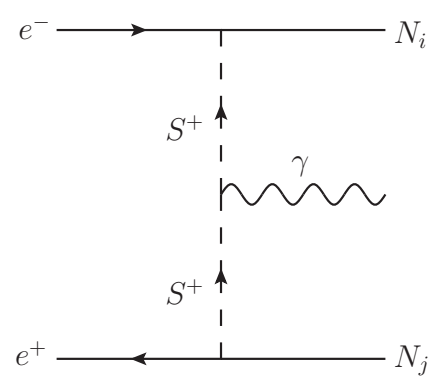

$(a)$

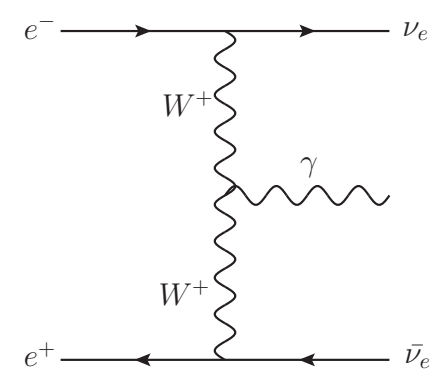

(b)

FIG. 7: The main Feynman diagram (a) [(b)] that contributes to the signal [background] for the process $e^{-} e^{+} \rightarrow \gamma+E_{\text {miss }}$.

The most interesting signature involves a pair of DM in the final state with one (or more) photon(s) irradiated from the intermediate charged scalar as depicted in Fig. 7. In this case, the background corresponds to a single (or multiple) photon(s) plus light neutrinos which can be reduced by applying the cut $E_{\gamma}>8 \mathrm{GeV}$ over the energy of the photon. Another potential signature is a pair production of charged scalars $S^{+} S^{-}$without or with a photon in the final state, as shown in Figs. 8 and 9, where $S^{ \pm}$decays into a RH neutrino and a charged lepton. In this 


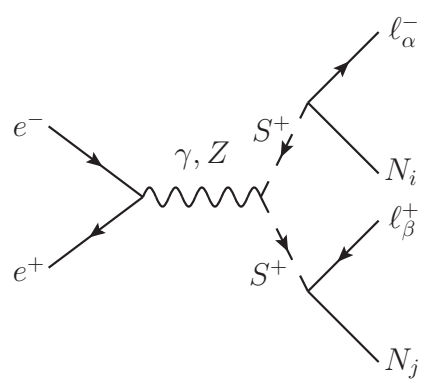

(a)

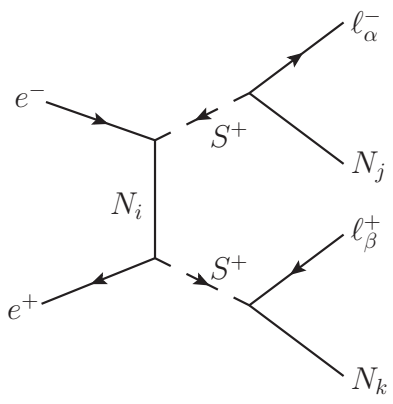

(b)

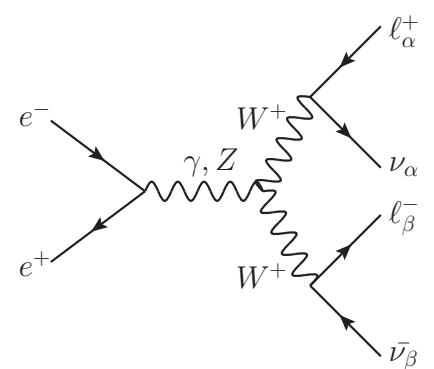

(c)

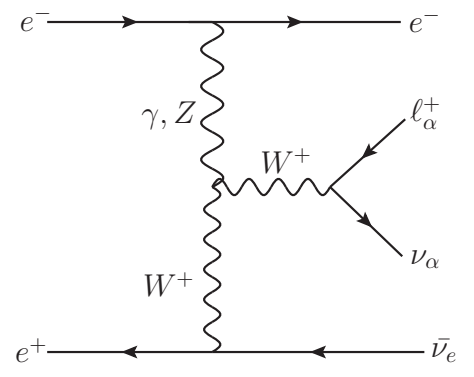

$(d)$

FIG. 8: The most important Feynman diagrams (a),(b) $[(\mathrm{c}),(\mathrm{d})]$ that contribute to the signal (background) for the process $e^{-} e^{+} \rightarrow S^{+} S^{-}$.

case, the background contributing to the signal comes from the process $e^{+} e^{-} \rightarrow W^{+} W^{-}$where each $W$ decays into a charged lepton and a light neutrino. Also, a same-sign pair of charged scalars can be produced in electron-electron collisions, as seen in Fig. 10, where a final state with same-sign leptons with missing energy can be observed. Therefore, in this study we consider the following processes:

$$
\begin{aligned}
& e^{-} e^{+} \rightarrow \gamma+E_{\text {miss }}, \\
& e^{-} e^{+} \rightarrow S^{+} S^{-} \rightarrow \ell_{\alpha}^{+} \ell_{\beta}^{-}+E_{m i s s}, \\
& e^{-} e^{-} \rightarrow S^{-} S^{-} \rightarrow \ell_{\alpha}^{-} \ell_{\beta}^{-}+E_{m i s s} \\
& e^{-} e^{+} \rightarrow \gamma+S^{+} S^{-} \rightarrow \gamma+\ell_{\alpha}^{+} \ell_{\beta}^{-}+E_{m i s s},
\end{aligned}
$$

and limit ourselves to a center-of-mass energy of $\sqrt{s}=500 \mathrm{GeV}$ and with a luminosity $L=$ $100 \mathrm{pb}^{-1}$. We generate three sets of benchmark points according to different values of the ratio $R_{1} \approx 1, R_{2} \approx 10^{-2}$, and $R_{3} \approx 10^{-4}$ which are in agreement with the observed DM relic density and respect the LFV constraints. The background processes (13) are due to the exchange of $W / Z / \gamma$ gauge bosons, and the corresponding Feynman diagrams can be similar to the ones for the signal. In Fig. 11, we show the cross section values and the corresponding significance at $L=100 \mathrm{pb}^{-1}$ for the processes $(13)$ as a function of the charged scalar mass before applying any cut. ${ }^{5}$

It is worth noting that $\sigma\left(e^{-} e^{+} \rightarrow S^{+} S^{-}\right)$and $\sigma\left(e^{-} e^{+} \rightarrow S^{+} S^{-}+\gamma\right)$ are dominated by the diagrams due to interactions (1) rather than the Drell-Yan diagrams. The interference contributions could be negative and can make the cross section smaller than the Drell-Yan one, as

${ }^{5}$ Except the cut $E_{\gamma}>8 \mathrm{GeV}$ and $\left|\cos \theta_{\gamma}\right|<0.998$ for the monophoton and $S^{-} S^{+}+\gamma$ channels. 


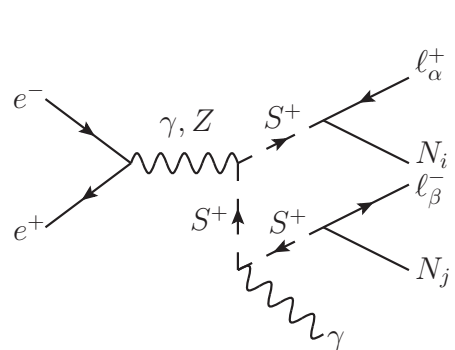

(a)

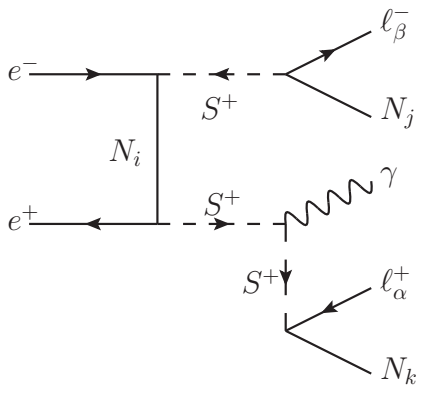

(b)

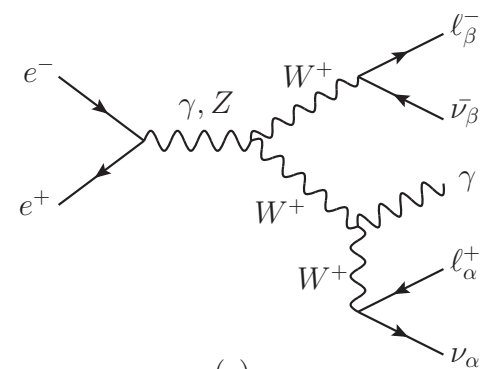

$(c)$

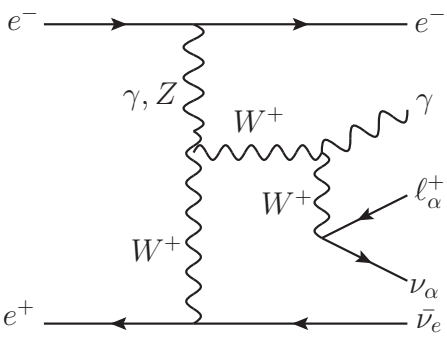

$(d)$

FIG. 9: The most important Feynman diagrams (a),(b) $[(\mathrm{c}),(\mathrm{d})]$ that contribute to the signal (background) for the process $e^{-} e^{+} \rightarrow S^{+} S^{-}+\gamma$.

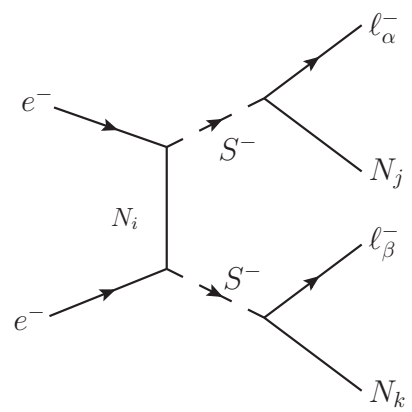

$(a)$

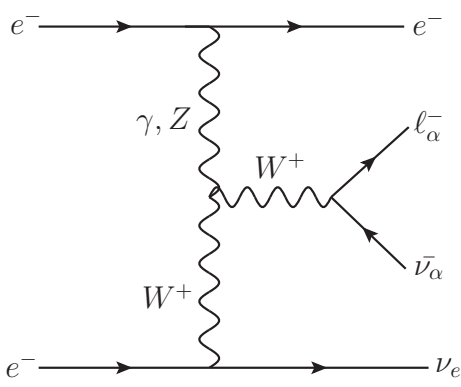

(b)

FIG. 10: The main Feynman diagram (a) [(b)] that contributes to the signal (background) for the process $e^{-} e^{-} \rightarrow S^{-} S^{-}$.

shown in Fig. 11 (left). As one can see, $\sigma\left(e^{-} e^{+} \rightarrow S^{+} S^{-}+\gamma\right)$ is about 100 times smaller than $\sigma\left(e^{-} e^{+} \rightarrow S^{+} S^{-}\right)$due to the $e^{2} / 4 \pi$ suppression from the coupling of the charged scalar to the photon. The production cross section of the same-sign charged scalars via an electron-electron collision is huge compared to the background, and hence the signal significance in this case could be large even for low luminosity. Therefore, this process is a clean and direct probe for $\mathrm{RH}$ neutrinos at the ILC.

\section{BENCHMARK ANALYSIS}

In this section, we consider three benchmark points with fine-tuning parameters $R_{1} \approx 1, R_{2} \approx$ $10^{-2}$, and $R_{3} \approx 10^{-4}$, respectively. As can be seen from Table- I, the choice of the ratios $R_{i}$ limits substantially our freedom of the model parameter space. Using CCALCHEP [24], we generate the 

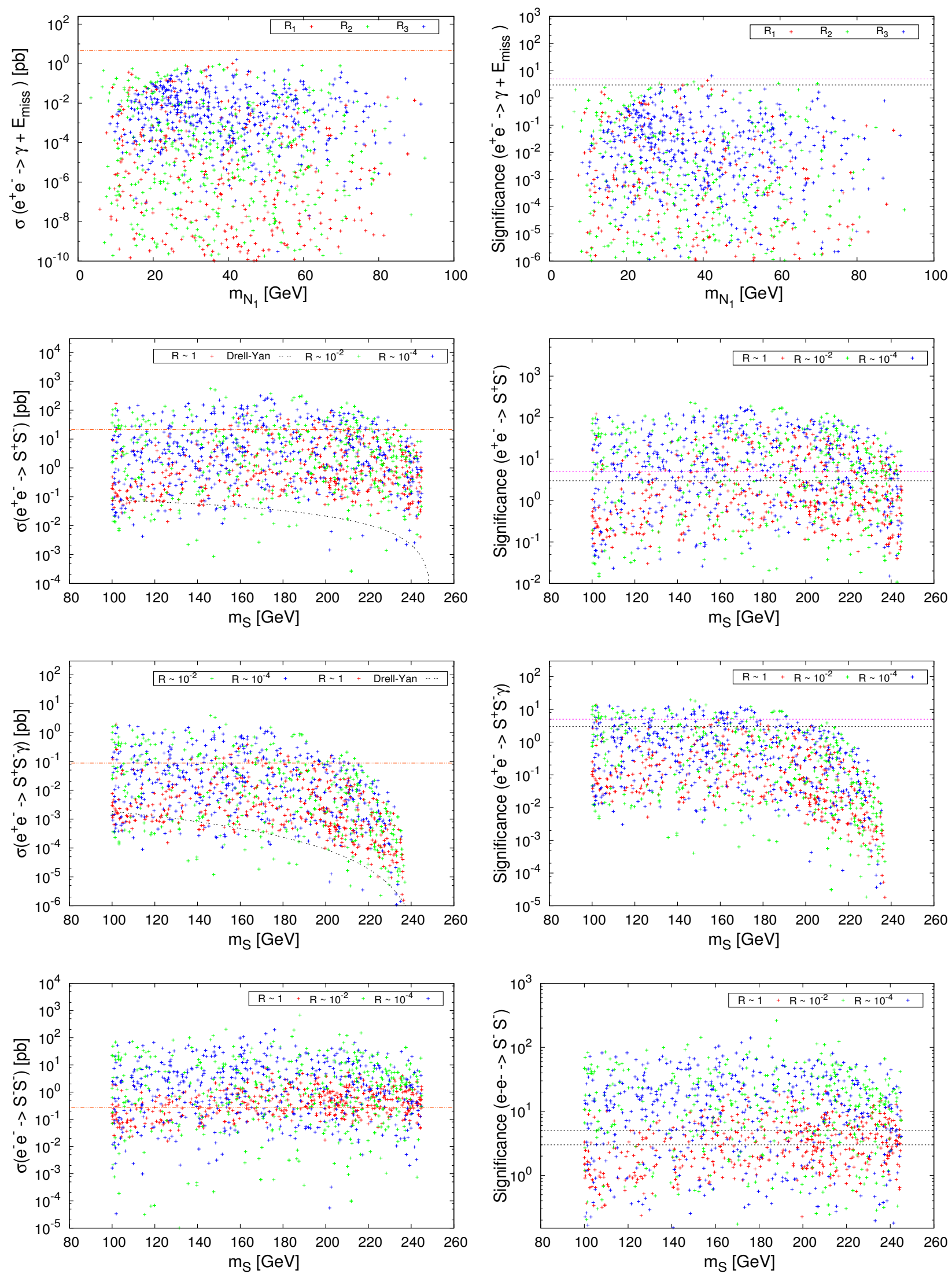

FIG. 11: The cross section values (left) and the corresponding significance values (right) at luminosity $100 \mathrm{pb}^{-1}$ in a function of $m_{S}$ for randomly chosen sets of parameters within three specific values of the fine-tuning parameter $R$. The red lines (left) represent the background value, and the dashed one represents the Drell-Yan contribution, and the dashed lines (right) represent $S=3,5$, respectively. 
distributions for different kinematic variables for both the signal and background of the processes $e^{-} e^{+} \rightarrow \gamma+E_{\text {miss }}, e^{-} e^{+} \rightarrow S^{-} S^{+}$, and $e^{-} e^{+} \rightarrow S^{-} S^{+}+\gamma$ at $500 \mathrm{GeV}$ center-of-mass energy. Then, we apply the kinematical cuts that optimize the signal detection over the background and estimate the corresponding signal significance for each process.

\begin{tabular}{cccc}
\hline Point & $B_{1}\left(R_{1}\right)$ & $B_{2}\left(R_{2}\right)$ & $B_{3}\left(R_{3}\right)$ \\
\hline$g_{1 e}$ & $(7.506+i 0.014) \times 10^{-1}$ & $(1.8284+i 0.103)$ & $(-0.103+i 0.201)$ \\
\hline$g_{2 e}$ & $(-0.26819-i 1.5758) \times 10^{-4}$ & $(1.543+i 3.004) \times 10^{-4}$ & $(0.654-i 2.616) \times 10^{-2}$ \\
\hline$g_{3 e}$ & $(-1.360-i 0.707)$ & $(0.313-i 0.549)$ & $(-0.869-i 0.878)$ \\
\hline$m_{S}(\mathrm{GeV})$ & 196.75 & 242.81 & 104.47 \\
\hline$m_{N_{1}}(\mathrm{GeV})$ & 25.788 & 43.764 & 38.306 \\
\hline$m_{N_{2}}(\mathrm{GeV})$ & 28.885 & 58.182 & 56.481 \\
\hline$m_{N_{3}}(\mathrm{GeV})$ & 36.274 & 67.511 & 72.440 \\
\hline
\end{tabular}

TABLE I: Three benchmark points selected from the parameter space of the model for a detailed analysis.

\section{A. The monophoton final state $\gamma+E_{\text {miss }}$}

First we use the distributions with precuts $E_{\gamma}>8 \mathrm{GeV}$ and $\left|\cos \theta_{\gamma}\right|<0.998$ and $E_{\text {miss }}>$ $100 \mathrm{GeV}$ and then deduce the cuts that reduce the contribution of the background relative to the signal. We find this can be achieved for the following cuts :

$$
8 \mathrm{GeV}<E_{\gamma}<300 \mathrm{GeV}, \quad\left|\cos \theta_{\gamma}\right|<0.998, \quad E_{\text {miss }}>300 \mathrm{GeV} .
$$

The distributions of the missing energy and the photon transverse momentum variables after applying the above cuts are shown in Fig. 12.
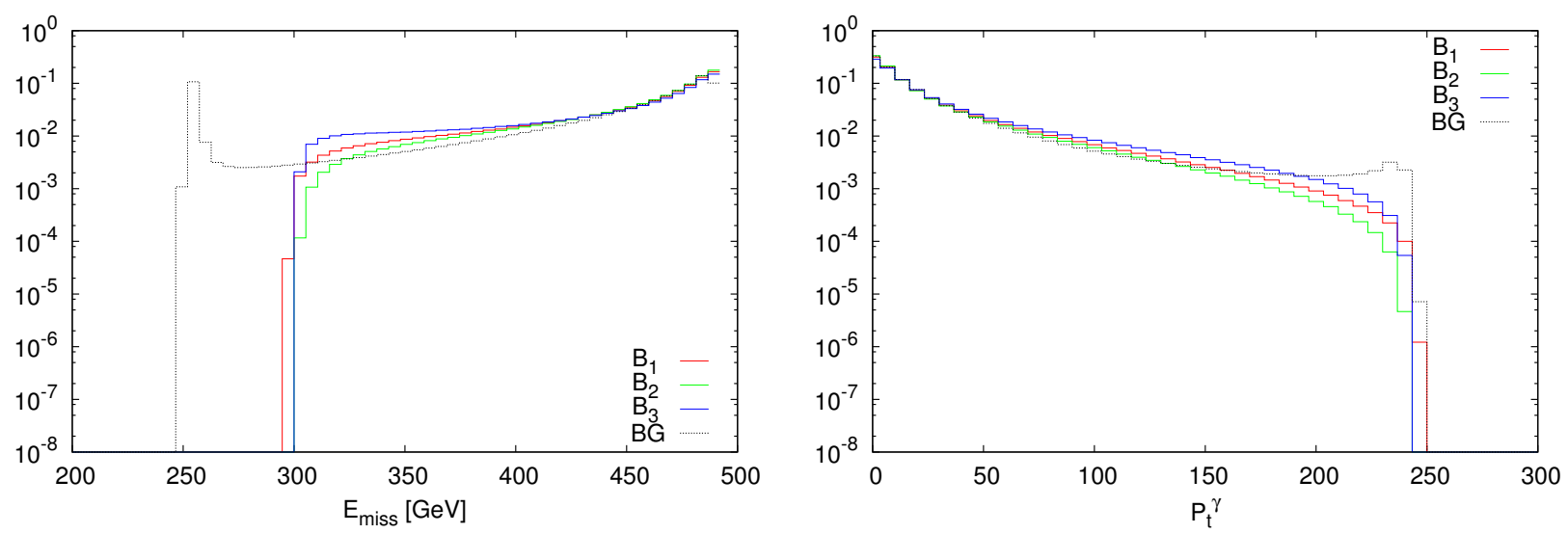

FIG. 12: The normalized distributions $E_{\text {miss }}$ and $p_{t}^{\gamma}$ of the process $e^{-} e^{-} \rightarrow \gamma+E_{\text {miss }}$ at $\sqrt{s}=500$ $\mathrm{GeV}$. The cuts used here are $E_{\gamma}>8 \mathrm{GeV}$ and $\left|\cos \theta_{\gamma}\right|<0.998$.

By varying the charged scalar mass, we show in Fig. 13 the signal significance at integrated luminosities $L=10,100$, and $500 \mathrm{fb}^{-1}$ for each benchmark point. 


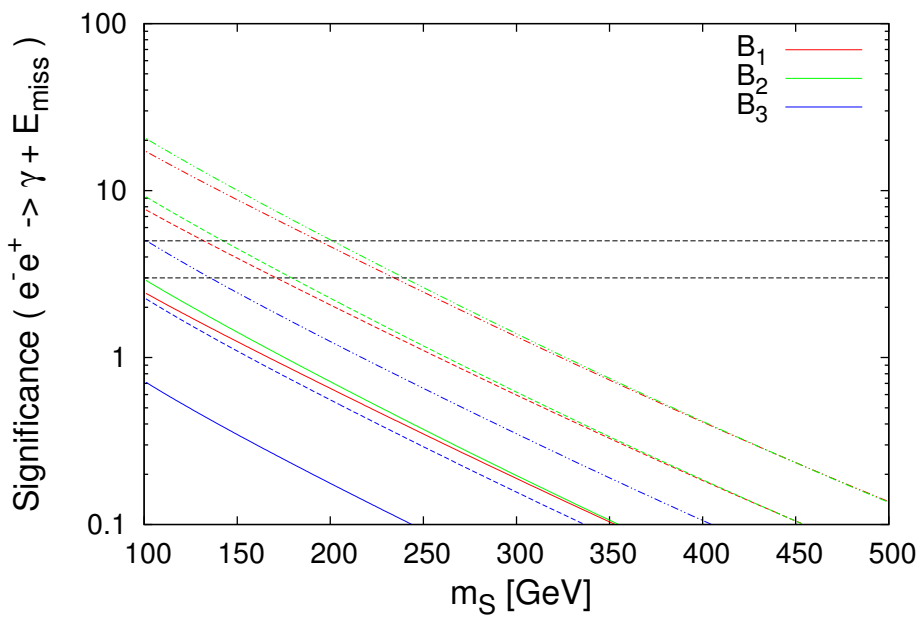

FIG. 13: The signal significance for the process $e^{-} e^{+} \rightarrow \gamma+E_{\text {miss }}$ as a function of $m_{S}$ for the chosen value of $g_{i \alpha}$ given in Table- I at an integrated luminosity of 10 (solid lines), 100 (dashed lines), and $500 \mathrm{fb}^{-1}$ (dash-dotted lines). The horizontal dashed lines correspond to a 3 and 5 sigma significance.

As can be seen, for charge scalars lighter than $200 \mathrm{GeV}$, the signal-to-background ratio could be larger than unity, which makes the RH neutrino signal detectable at the ILC for a luminosity of a few hundred $\mathrm{fb}^{-1}$ whatever the fine-tuning parameter value. However, for charged scalars heavier than $300 \mathrm{GeV}$, it requires a very high luminosity for the signal to be detected with a 5 sigma significance or larger.

\section{B. Final state $S^{+} S^{-}(\gamma)$}

Similar to the monophoton analysis, we generate different distributions for the relevant kinematic variables and then select the following cuts that maximize the signal-to-background ratio:

$$
\text { Final state } S^{+} S^{-}:\left\{\begin{array}{l}
M_{\ell^{+}, \ell^{-}}<300 \mathrm{GeV}, 150 \mathrm{GeV}<E_{\text {miss }}<420 \mathrm{GeV} \\
30 \mathrm{GeV}<E^{\ell}<180 \mathrm{GeV}, p_{t}^{\ell}<170 \mathrm{GeV}
\end{array}\right.
$$

and

$$
\text { Final state } S^{+} S^{-} \gamma:\left\{\begin{array}{l}
M_{\ell^{+}, \ell^{-}}<300 \mathrm{GeV}, 150 \mathrm{GeV}<E_{\text {miss }}<400 \mathrm{GeV} \\
30 \mathrm{GeV}<E^{\ell}<170 \mathrm{GeV}, p_{t}^{\ell}<170 \mathrm{GeV} \\
\left|\cos \left(\theta_{\gamma}\right)\right|<0.5,8 \mathrm{GeV}<E^{\gamma}<120 \mathrm{GeV}, p_{t}^{\gamma}<110 \mathrm{GeV} .
\end{array}\right.
$$

The relevant normalized kinematical distributions for the processes $e^{-} e^{+} \rightarrow S^{-} S^{+}$and $e^{-} e^{+} \rightarrow$ $S^{-} S^{+} \gamma$ with the above cuts applied, are presented in Figs. 14 and 15 , respectively.

In Fig. 16, we show the significance for these processes as a function of $m_{S}$ for the three considered benchmark points. We see that for the production of a pair of charged scalars without a photon in the final state the signal-to-background ratio can be very large for $m_{S}<220 \mathrm{GeV}$ even at a very low integrated luminosity (about $0.5 \mathrm{fb}^{-1}$ ), whereas for larger masses the signal detection requires a huge luminosity. Hence, for charged scalars lighter than about $220 \mathrm{GeV}$, the process $e^{-} e^{+} \rightarrow S^{-} S^{+}$can be easily testable at the ILC. Concerning the final state with a 

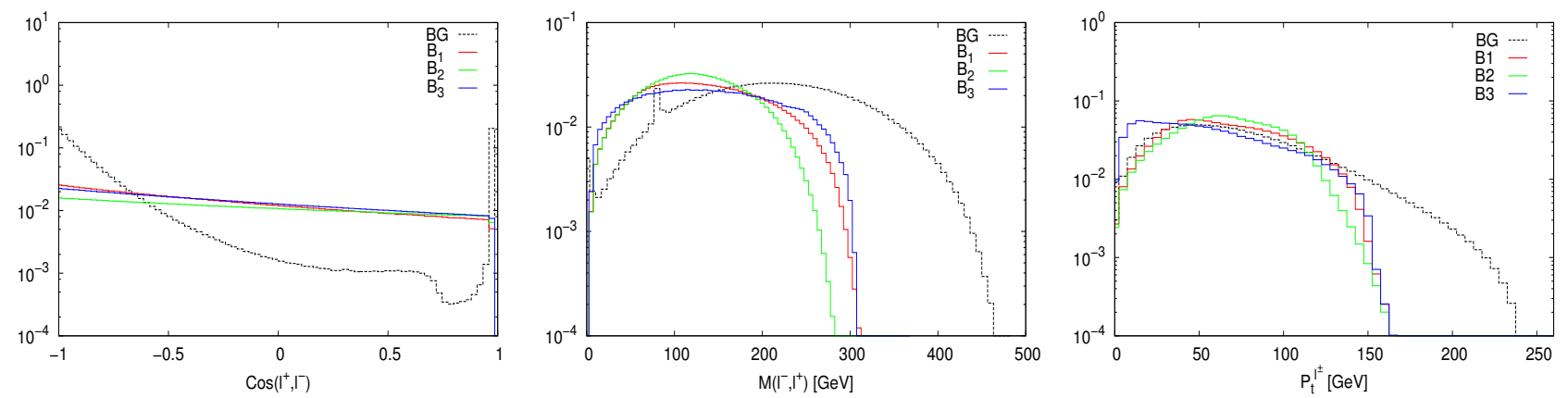

FIG. 14: The angular, invariant mass, and transverse momentum normalized distributions for charged leptons of process $e^{-} e^{+} \rightarrow S^{-} S^{+}$at $\sqrt{s}=500 \mathrm{GeV}$.
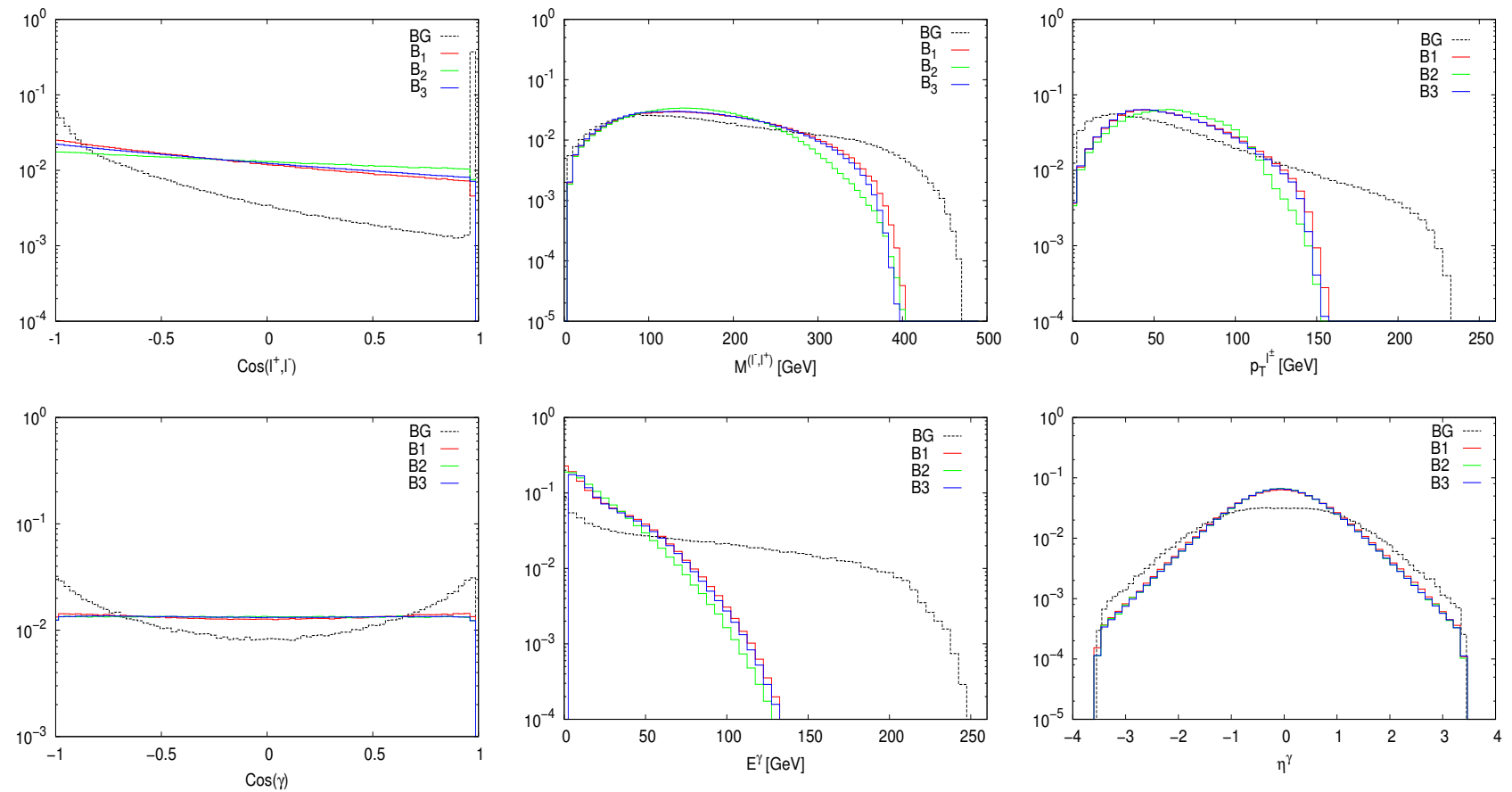

FIG. 15: Different normalized distributions of the process $e^{-} e^{+} \rightarrow S^{-} S^{+}+\gamma$ at $\sqrt{s}=500 \mathrm{GeV}$. At the top from left to right, the angular normalized distribution $\cos \left(\ell^{+}, \ell^{-}\right)$, invariant mass $M_{\ell^{+}, \ell^{-}}$, and the charged lepton transverse momentum $p_{t}^{\ell^{-}}$. At the bottom from left to right, the angular, the energy, and the rapidity normalized distributions of the photon, respectively.

photon, for a luminosity of few tens of $\mathrm{fb}^{-1}$, the significance is large for a charged scalar mass less than $150 \mathrm{GeV}$ and between 180 and $220 \mathrm{GeV}$.

\section{Analysis with polarized beams}

Using polarized electron or positron beams, which will be available at linear colliders such as the ILC, is an additional feature that allows the improvement of the detection of the signal of the considered processes. Indeed, for the processes we are considering, the interactions involve electrons (positrons) with only right- (left-) handed chirality, and a polarized beam can lead to an increase in the signal-to-background ratio. The ILC plans a longitudinal polarization 

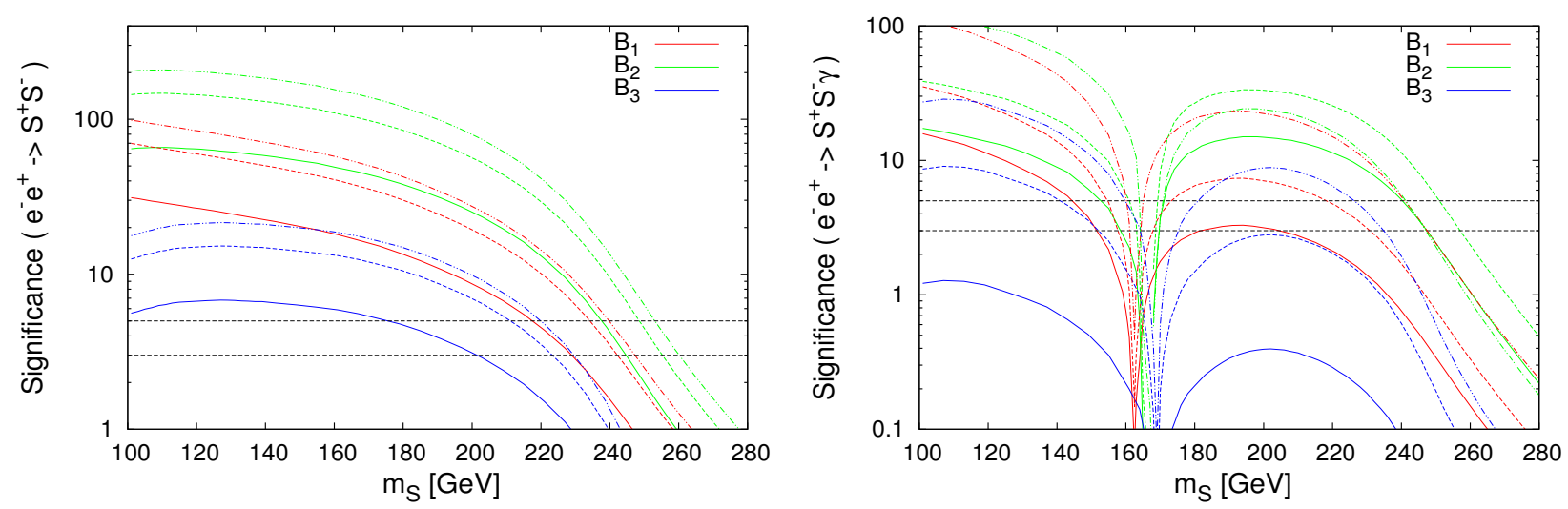

FIG. 16: The signal significance for the processes $e^{-} e^{+} \rightarrow S^{-} S^{+}$(left) and $e^{-} e^{+} \rightarrow S^{-} S^{+}+\gamma$ (right) as a function of $m_{S}$ for the values of $g_{i \alpha}$ given in Table- $\mathbb{1}$ at an integrated luminosity of 1 (solid lines), 5 (dashed lines), and $10 \mathrm{fb}^{-1}$ (dash-dotted lines) for $S^{-} S^{+}$and an integrated luminosity of 10 (solid lines), 50 (dashed lines) and $100 \mathrm{fb}^{-1}$ (dash-dotted lines) for $S^{-} S^{+}+\gamma$. The horizontal dashed lines correspond to a 3 and 5 sigma significance. For the values $m_{S}>250 \mathrm{GeV}$, the charged scalars is off shell.

of $80 \%\left(\left|P\left(e^{-}\right)\right|<0.8\right)$ for the electron beam and 30\% $\left(\left|P\left(e^{+}\right)\right|<0.3\right)$ for the positron beam with the possibility to upgrade to $60 \%$. Here, we reanalyze the processes discussed earlier by considering polarized beams as $P\left(e^{-}, e^{+}\right)=[+0.8,-0.3]$ while applying the same cuts used previously.

In Tables II, IV, we present the number of events of the signal and the background for the three benchmarks using different values of the electron and positron polarizations. We see clearly that with polarized beams the number of background events gets reduced by $86 \%$ and the signal increased by $130 \%$, and thus a substantial improvement of the significance for every process does exist. In particular, the process $e^{+} e^{-} \rightarrow S^{+} S^{-}+\gamma$ can be observed at a low luminosity of order of $\mathrm{fb}^{-1}$ for partially polarized electron and positron beams, whereas it requires a large luminosity value without polarized beams.

\begin{tabular}{|c|c|c|c|c|c|c|}
\hline$P\left(e^{-}, e^{+}\right)$ & $N_{B G}$ & $\mathrm{BP}$ & $N_{S}$ & $S_{10}$ & $S_{100}$ & $S_{500}$ \\
\hline \hline \multirow{3}{*}[0,0]{} & \multirow{4}{*}{46652} & $B_{1}$ & 172.2 & 0.80 & 2.516 & 5.63 \\
\cline { 3 - 7 } & $B_{2}$ & 122.2 & 0.565 & 1.79 & 3.99 \\
\hline & & $B_{3}$ & 130.1 & 0.61 & 1.90 & 4.25 \\
\hline \multirow{4}{*}[+0.8,-0.3]{} & \multirow{3}{*}{6541} & $B_{1}$ & 396.06 & 4.75 & 15.04 & 33.62 \\
\cline { 3 - 7 } & $B_{2}$ & 283.5 & 3.43 & 10.85 & 24.27 \\
\cline { 3 - 7 } & & $B_{3}$ & 299.23 & 3.62 & 11.44 & 25.58 \\
\hline
\end{tabular}

TABLE II: The background $\left(N_{B G}\right)$ and signal $\left(N_{S}\right)$ number of events for the process $e^{-} e^{+} \rightarrow \gamma+E_{\text {miss }}$ that corresponds to the integrated luminosity values $\mathrm{L}=10 \mathrm{fb}^{-1}$ for the three chosen benchmark points (Table-I), with or without polarized beams, within the cuts given in (14); the significance values $S_{10}$, $S_{100}$ and $S_{500}$ correspond to the three integrated luminosity values $L=10,100$, and $500 \mathrm{fb}^{-1}$.

In order to see the effect of the polarization on the signal, we present in Fig. 17 the significance for $P\left(e^{-}, e^{+}\right)=[0,0]$ and $P\left(e^{-}, e^{+}\right)=[+0.8,-0.3]$, for the benchmark point $B_{3}$ as a func- 


\begin{tabular}{|c|c|c|c|c|c|c|}
\hline$P\left(e^{-}, e^{+}\right)$ & $N_{B G}$ & $\mathrm{BP}$ & $N_{S}$ & $S_{0.1}$ & $S_{0.5}$ & $S_{1}$ \\
\hline \multirow{3}{*}[0,0]{} & \multirow{3}{*}{212036} & $B_{1}$ & 11312 & 2.39 & 5.35 & 7.57 \\
\hline & & $B_{2}$ & 7231 & 1.54 & 3.45 & 4.88 \\
\hline & & $B_{3}$ & 10660 & 2.26 & 5.05 & 7.14 \\
\hline \multirow{3}{*}[+0.8,-0.3]{} & \multirow{3}{*}{122397} & $B_{1}$ & 25904 & 6.73 & 15.04 & 21.27 \\
\hline & & $B_{2}$ & 17138 & 4.59 & 10.26 & 14.51 \\
\hline & & $B_{3}$ & 24625 & 6.42 & 14.36 & 20.31 \\
\hline
\end{tabular}

TABLE III: The background $\left(N_{B G}\right)$ and signal $\left(N_{S}\right)$ number of events for the process $e^{-} e^{+} \rightarrow S^{-} S^{+}$ that corresponds to the integrated luminosity values $\mathrm{L}=10 \mathrm{fb}^{-1}$ for the three chosen benchmark points (Table-I), with or without polarized beams, within the cuts given in (15); the significance values $S_{0.1}$, $S_{0.5}$, and $S_{1}$ correspond to the three integrated luminosity values $L=0.1,0.5$, and $1 \mathrm{fb}^{-1}$.

\begin{tabular}{|c|c|c|c|c|c|c|}
\hline$P\left(e^{-}, e^{+}\right)$ & $N_{B G}$ & $\mathrm{BP}$ & $N_{S}$ & $S_{10}$ & $S_{50}$ & $S_{100}$ \\
\hline \hline \multirow{4}{*}[0,0]{} & \multirow{3}{*}{876.39} & $B_{1}$ & 26.56 & 0.88 & 1.98 & 2.79 \\
\cline { 3 - 7 } & $B_{2}$ & 10.52 & 0.35 & 0.79 & 1.12 \\
\hline & & $B_{3}$ & 66.10 & 2.15 & 4.81 & 6.81 \\
\hline \multirow{4}{*}[+0.8,-0.3]{} & \multirow{3}{*}{123.20} & $B_{1}$ & 61.48 & 4.52 & 10.11 & 14.30 \\
\cline { 3 - 7 } & & $B_{2}$ & 24.24 & 2.00 & 4.46 & 6.31 \\
\hline & $B_{3}$ & 150.05 & 9.08 & 20.30 & 28.70 \\
\hline
\end{tabular}

TABLE IV: The background $\left(N_{B G}\right)$ and signal $\left(N_{S}\right)$ number of events for the process $e^{-} e^{+} \rightarrow S^{-} S^{+}+\gamma$ that corresponds to the integrated luminosity values $\mathrm{L}=10 \mathrm{fb}^{-1}$ for the three chosen benchmark points (Table-I), with or without polarized beams, within the cuts given in (15); the significance values $S_{10}$, $S_{50}$ and $S_{100}$ correspond to the three integrated luminosity values $L=10,50$, and $100 \mathrm{fb}^{-1}$.

tion of luminosity. We clearly see that the signal over the background gets improved significantly. For $P\left(e^{-}, e^{+}\right)=[-0.8,+0.3],\left[N_{B G}, N_{S}\right]$ get modified by $[+189 \%,-88 \%]$. In the case where only the electron beam is polarized with $P\left(e^{-}\right)=+0.8(-0.8),\left[N_{B G}, N_{S}\right]$ are changed by $[-78 \%,+80 \%]([+85 \%,-78 \%])$. On the other hand, where only the positron beam is polarized with $P\left(e^{+}\right)=+0.3(-0.3),\left[N_{B G}, N_{S}\right]$ are changed by $[+63 \%,-43 \%]([-59 \%,+62 \%])$.

\section{CONCLUSION}

In this work, we investigated some of type of interactions that are part of a generic class of radiative neutrino mass models which involve a heavy right-handed neutrino coupled to a $\mathrm{RH}$ charged lepton and singlet charged scalar. We find that in order to be consistent with the current experimental bounds on the LFV processes such as $\ell_{\alpha} \rightarrow \ell_{\beta}+\gamma$ requires the coupling of the charged leptons with the RH neutrinos to be suppressed which can be in conflict with the observed DM relic density. For that we defined a fine-tuning parameter $R$ that measures how small the couplings have to be to satisfy both the DM constraint and the LFV bounds. Hence, in our analysis we consider three sets of benchmark points that avoid the experimental limits on the LFV branching fraction with $R_{1} \sim 1$ (without fine-tuning), $R_{2} \sim 10^{-2}$ (moderately fine-tuned), and $R_{2} \sim 10^{-4}$ (highly fine-tuned). We also used the data from LEP-II on a monophoton plus 

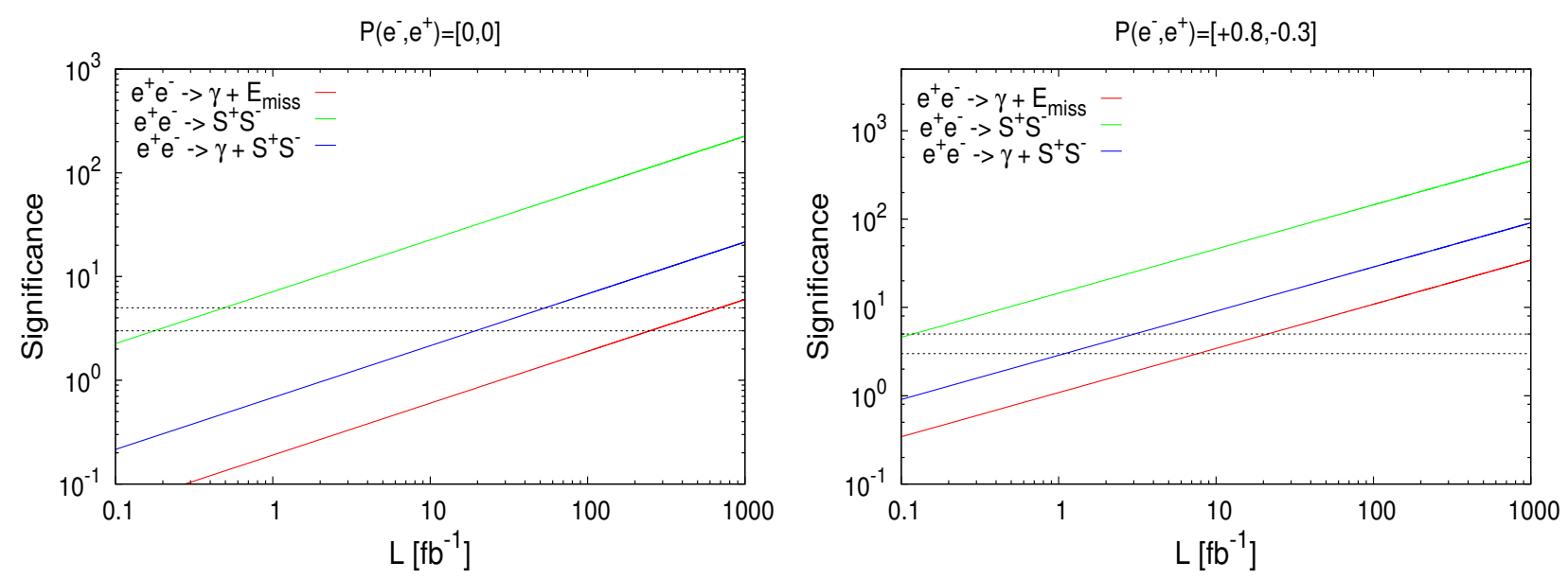

FIG. 17: The signal significance versus the luminosity for the three different signatures studied, without polarization at left and with polarization of $P\left(e^{-}, e^{+}\right)=[+0.8,-0.3]$ at right. The two horizontal dashed lines in each figure indicate the corresponding significance values for $S=3$ and $S=5$, respectively.

missing energy to constrain the model space parameter and derived an approximate analytical bound on the coupling of the right-handed charged lepton to the RH neutrino and charged scalar. Interestingly, we found that, even before applying kinematical cuts, there are points for which the cross sections of the processes $e^{-} e^{+} \rightarrow \gamma+E_{\text {miss }}, S^{-} S^{+}, S^{-} S^{+}+\gamma$ are much larger than the corresponding background by more than one order of magnitude. Thus, the future lepton colliders will be able to probe a significant fraction of the parameter space of this class of radiative neutrino mass models even at a low luminosity.

Among the scanned benchmark points with comparable $\mathrm{RH}$ neutrino masses, we have chosen three of them with $R_{1} \approx 1, R_{2} \approx 10^{-2}$, and $R_{3} \approx 10^{-4}$ to see the effect of the fine-tuning. Then we applied the appropriate cuts on the kinematical variables to reduce the background. We found that the signal can be seen at an integrated luminosity of $\mathcal{O}\left(100 \mathrm{fb}^{-1}\right), \mathcal{O}\left(\mathrm{fb}^{-1}\right)$, and $\mathcal{O}\left(10 \mathrm{fb}^{-1}\right)$ for the processes $\gamma+E_{\text {miss }}, S^{+} S^{-}$, and $S^{+} S^{-}+\gamma$, respectively, whereas, one cannot disentangle the effect of the fine-tuning. For these values of the luminosity, the charged scalar should be lighter than $220 \mathrm{GeV}$.

Finally, since the interactions considered in this work involve exclusively the right-handed charged lepton, we studied the effect of polarized $e^{-} / e^{+}$beams, as will be used at future linear colliders such as the ILC or CLIC. We have shown that the signal-to-background ratio gets enhanced by a factor of more than 5 for the polarization $P\left(e^{-}, e^{+}\right)=[+0.8,-0.3]$. Thus, with the use of polarized beams, a large parameter space of this class of neutrino radiative models can be probed for with a low luminosity via different processes at the starting of the planned ILC and CLIC colliders.

\section{Appendix A: Loop functions}

Here, we give the loop functions used in Sec. II, which are given by 


$$
\begin{gathered}
F(x)=\frac{1-6 x+3 x^{2}+2 x^{3}-6 x^{2} \log x}{6(1-x)^{4}}, \\
G(x)=\frac{2-9 x+18 x^{2}-11 x^{3}+6 x^{3} \log x}{6(1-x)^{4}} \\
D_{1}(x, y)=-\frac{1}{(1-x)(1-y)}-\frac{x^{2} \log x}{(1-x)^{2}(x-y)}-\frac{y^{2} \log y}{(1-y)^{2}(y-x)}, \\
D_{2}(x, y)=-\frac{1}{(1-x)(1-y)}-\frac{x \log x}{(1-x)^{2}(x-y)}-\frac{y \log y}{(1-y)^{2}(y-x)} .
\end{gathered}
$$

These loop functions does not diverge and behave as follows near critical points:

$$
\begin{gathered}
D_{1}(x, x)=\frac{-1+x^{2}-2 x \log x}{(1-x)^{3}}, \\
D_{2}(x, x)=\frac{-2+2 x-(1+x) \log x}{(1-x)^{3}}, \\
D_{1}(x, 1)=D_{1}(1, x)=\frac{-1+4 x-3 x^{2}+2 x^{2} \log x}{2(1-x)^{3}}, \\
D_{2}(x, 1)=D_{2}(1, x)=\frac{1-x^{2}+2 x \log x}{2(1-x)^{3}},
\end{gathered}
$$

and

$$
F(1)=\frac{1}{10}, \quad G(1)=\frac{1}{4}, \quad D_{1}(1,1)=\frac{1}{3}, \quad D_{2}(1,1)=\frac{1}{6} .
$$

[1] M. Gell-Mann, P. Ramond, and R. Slansky, in Supergravity, edited by P. van Nieuwenhuizen and D. Z. Freedman (North-Holland, Amsterdam, 1979), p. 315; T. Yanagida, in Proceedings of the Workshop on the Unified Theory and the Baryon Number in the Universe, edited by O. Sawada and A. Sugamoto, KEK Report No. 79-18 (Tsukuba, Japan, 1979), p. 95; R. N. Mohapatra and G. Senjanovic, Phys. Rev. Lett. 44, 912 (1980).

[2] W. Y. Keung and G. Senjanovic, Phys. Rev. Lett. 50, 1427 (1983); T. Han and B. Zhang, Phys. Rev. Lett. 97, 171804 (2006); A. Atre, T. Han, S. Pascoli, and B. Zhang, J. High Energy Phys. 05 (2009) 030; C. Y. Chen and P. S. B. Dev, Phys. Rev. D 85, 093018 (2012); C. Y. Chen, P. S. B. Dev, and R. N. Mohapatra, Phys. Rev. D 88, 033014 (2013); P. S. B. Dev, A. Pilaftsis, and U. K. Yang, Phys. Rev. Lett. 112, 081801 (2014); D. Alva, T. Han, and R. Ruiz, J. High Energy Phys. 02 (2015) 072; F. F. Deppisch, P. S. Bhupal Dev, and A. Pilaftsis, New J. Phys. 17, 075019 (2015); A. Das, P. Konar, and S. Majhi, J. High Energy Phys. 06 (2016) 019; S. Antusch, E. Cazzato, and O. Fischer, arXiv:1612.02728; A. Das and N. Okada, arXiv:1702.04668.

[3] A. Zee, Phys. Lett. 161B, 141 (1985).

[4] A. Zee, Nucl. Phys. B 264, 99 (1986); K. S. Babu, Phys. Lett. B 203, 132 (1988).

[5] E. Ma, Phys. Rev. D73,077301(2006) .

[6] L. M. Krauss, S. Nasri, and M. Trodden, Phys. Rev. D67,085002(2003). 
[7] M. Aoki, S. Kanemura, and O. Seto, Phys. Rev. Lett. 102, 051805 (2009) ; Phys. Rev. D 80, 033007 (2009) .

[8] A. Ahriche, C. S. Chen, K. L. McDonald, and S. Nasri, Phys. Rev. D 90, 015024 (2014) ; A. Ahriche, K. L. McDonald and S. Nasri, J. High Energy Phys. 10 (2014) 167; 02 (2016) 038.

[9] A. Ahriche, S. Nasri, and R. Soualah, Phys. Rev. D 89, 095010 (2014) ; C. Guella, D. Cherigui, A. Ahriche, S. Nasri, and R. Soualah, Phys. Rev. D 93, 095022 (2016) ; D. Cherigui, C. Guella, A. Ahriche, and S. Nasri, Phys. Lett. B 762, 225 (2016) .

[10] J. Adam et al. (MEG Collaboration), Phys. Rev. Lett. 110, 201801 (2013) .

[11] U. Bellgardt et al. (SINDRUM Collaboration), Nucl. Phys. B 299, 1 (1988).

[12] B. Aubert et al. (BABAR Collaboration), Phys. Rev. Lett. 104, 021802 (2010) .

[13] K. Hayasaka et al., Phys. Lett. B 687, 139 (2010) .

[14] P. Achard et al. (L3 Collaboration), Phys. Lett. B 587, 16 (2004) .

[15] H. Okada and K. Yagyu, Phys. Rev. D 93, 013004 (2016) ; L. G. Jin, R. Tang, and F. Zhang, Phys. Lett. B 741, 163 (2015) ; K. Cheung, T. Nomura, and H. Okada, Phys. Rev. D 95, 015026 (2017); S. Baek, H. Okada, and T. Toma, J. Cosmol. Astropart, Phys. 06 (2014) 027 ; S. Kashiwase, H. Okada, Y. Orikasa, and T. Toma, Int. J. Mod. Phys. A 31, 1650121 (2016) ; S. Kanemura, K. Nishiwaki, H. Okada, Y. Orikasa, S. C. Park, and R. Watanabe, Prog. Theor. Exp. Phys. 2016 , 123B04 (2016) ; S. Kanemura, O. Seto, and T. Shimomura, Phys. Rev. D 84, 016004 (2011).

[16] A. Ahriche, K. L. McDonald, S. Nasri, and T. Toma, Phys. Lett. B 746, 430 (2015) .

[17] T. Toma and A. Vicente, J. High Energy Phys. 01 (2014) 160 ; J. Hisano, T. Moroi, K. Tobe, and M. Yamaguchi, Phys. Rev. D 53, 2442 (1996) .

[18] K. A. Olive et al. (Particle Data Group), Chin. Phys. C 38, 090001 (2014).

[19] G. Venanzoni (Fermilab E989 Collaboration), Nucl. Part. Phys. Proc. 273-275, 584 (2016); T. Mibe (J-PARC g-2 Collaboration), Chin. Phys. C 34, 745 (2010).

[20] V. Tello, M. Nemevsek, F. Nesti, G. Senjanovic, and F. Vissani, Phys. Rev. Lett. 106, 151801 (2011) .

[21] A. Ahriche, K. L. McDonald, and S. Nasri, J. High Energy Phys. 06 (2016) 182.

[22] A. Ahriche and S. Nasri, J. High Energy Phys. 07 (2013) 035.

[23] A. Semenov, Comput. Phys. Commun. 201, 167 (2016) .

[24] A. Belyaev, N. D. Christensen and A. Pukhov, Comput. Phys. Commun. 184, 1729 (2013) .

[25] H. Baer et al., arXiv:1306.6352.

[26] M. J. Boland et al. (CLIC and CLICdp Collaborations), arXiv:1608.07537. 